Gut, 1987, 28, 52-55

\title{
Effect of chronic ethanol ingestion on enterocyte turnover in rat small intestine
}

\author{
R MAZZANTI AND W J JENKINS \\ From the Department of Medicine, Royal Free Hospital School of Medicine, Rowland Hill Street, London
}

SUMMARY Whether chronic ethanol ingestion significantly damages the small intestine remains controversial. To clarify this we have analysed the morphology of the small intestinal epithelium and quantified its renewal in chronically ethanol fed rats. Twenty adult male rats were pair fed for 28 days a nutritionally adequate liquid diet containing either ethanol as $36 \%$ of total calories or an isocaloric diet in which fat substituted for ethanol. Crypt cell production rate was determined in the jejunum and ileum by the metaphase arrest method. Weight gain and small intestinal morphology were similar in ethanol fed and control rats, but enterocyte turnover was significantly reduced in the jejunum $(\mathrm{p}<0 \cdot 05)$ and ileum $(\mathrm{p}<() \cdot() 1)$ of the ethanol fed rats. This effect of ethanol on the small intestine is probably systemic rather than local, because the changes in jejunum and ileum were similar, and it may contribute to the development of malnutrition in chronic alcoholics.

A variety of changes in intestinal function have been reported after acute or habitual alcohol consumption. ${ }^{1}$ Impaired absorption of vitamins, ${ }^{2-5}$ sugars, ${ }^{67}$ and amino acids ${ }^{89}$ has been shown in the presence of ethanol, and the chronic consumption of ethanol is often associated with malnutrition and diarrhoea, but whether these are caused by the alcohol itself, or may be due in part or whole to associated nutritional deficiencies remains unknown. ${ }^{1}$

Acute exposure of the small intestine to high concentrations of ethanol damages the mucosa causing haemorrhages, erosions, and blebs. ${ }^{10}$ After chronic ethanol consumption, however, the small intestinal mucosa has been reported to be histologically normal both in $\operatorname{man}^{11}$ and experimental animals, ${ }^{12}$ although shortened villi have also been described. ${ }^{10}$ Increased passive permeability of the small intestine has recently been described in animals after acute exposure to ethanol ${ }^{13}{ }^{14}$ and in chronic alcoholics. ${ }^{15}$ Because the integrity of the gut mucosa is dependent on the continual renewal of enterocytes, it is possible that ethanol may alter small intestinal structure and function by affecting enterocyte turnover. Accordingly we have investigated epithelial replacement in the small intestines of chronically ethanol fed rats.

Address for correspondence: Dr W J Jenkins. DHSS Medicines Division. Market Towers. 1 Nine Elms Lane, London. SW8 !NO. Received for publication 2 May 1986.

\section{Methods}

\section{ANIMALS}

Twenty adult male Sprague-Dawley rats, which initially weighed between $210 \mathrm{~g}$ and $310 \mathrm{~g}$, were maintained in single cages on a 12 hour light-dark cycle at $21 \pm 1^{\circ} \mathrm{C}$ room temperature. They were pair fed for 28 days nutritionally adequate diets similar to one described previously ${ }^{16}$ containing Complan, casein, glucose, corn oil and Orovite 7 , but in different proportions to provide $36 \%, 34 \%$, and $18 \%$ of the total calories as fat, glucose, and protein respectively in the control diet. The ethanol fed rats received a similar diet but ethanol $50 \mathrm{~g} / \mathrm{l}$ was substituted for fat to provide $36 \%$ of the total calories. The feeding bottles were renewed daily.

On day 28 at 930 am the rats were injected with vincristine sulphate $1 \mathrm{mg} / \mathrm{kg}$ body weight ip to produce metaphase arrest, and killed at intervals by cervical dislocation after very light ether anaesthesia. Immediately after death the whole small intestine was excised and measured under light traction while it was immersed in a saline buffer $\mathrm{pH} 7.4$ at $4^{\circ} \mathrm{C}$. Samples were taken from two sites at $15 \%$ and at $85 \%$ of the total length from the pylorus.

\section{HISTOLOGICAL AND MORPHOMETRIC}

EXAMINATION OF THE SMALL INTESTINE

Samples from the small intestines were fixed in $10 \%$ formalin and stained for histological examination in 
haematoxylin and eosin. Morphometric measurements were carried out in each sample by measuring the depth of 10 cyrpts, in which the bottom and neck were clearly visible, and the height of 10 well orientated villi. Samples were coded before examination by an observer, who did not know from which animal they had been taken.

MEASUREMENT OF CRYPT CELL PRODUCTION RATE (CCPR)

Crypt cell production rate was measured by the metaphase arrest (stathmokinetic) method. ${ }^{17}$ Rats were killed at approximately 30 minute intervals from 30-165 min after the intraperitoneal injection of vincristine sulphate. Samples from the small intestine were immediately fixed in Carnoy's solution for four to six hours and stored in $70 \%$ ethanol. On the day of reading they were passed through $50 \%$ ethanol and then $25 \%$ ethanol for 15 minutes in each solution, and then hydrolysed in $1 \mathrm{~m} \mathrm{HCl}$ for six minutes at $60^{\circ} \mathrm{C}$. They were stained in fresh Schiff's reagent for one hour. After rinsing in $45 \%$ acetic acid several crypts per slide were separated by microdissection using mounted needles and a dissecting microscope. The crypts were squashed in $45 \%$ acetic acid beneath a coverslip, and the metaphases per crypt counted under a $40 \mathrm{x}$ microscope objective. For each sample at least 10 crypts were counted. Each crypt came from a slightly different area of the tissue sample. Before counting the samples had been coded so that they were counted 'blind'. After decoding the results were plotted (Fig. 1), and the slope of the metaphase accumulation line and its standard error was calculated by linear regression. The slope of the line is the rate of entry of cells into mitosis, or the cell birth rate. Slopes were compared by a $t$ test, where the $t$ value is the difference between the two slopes divided by the square root of the sum of the squares of the standard errors.

CRYPT: VILLUS RATIO

The crypt: villus $(\mathrm{C} / \mathrm{V})$ ratio was determined in larger pieces of the tissue samples stained for CCPR measurement. The serosa and muscularis were removed using mounted needles. By selectively focusing on the bases of the villi and the necks of the crypts the number per field were counted using a microscope with a $10 \times 10$ grid in the eyepiece.

Net villus influx was calculated by multiplying CCPR by $\mathrm{C} / \mathrm{V}$ ratio.

\section{Results}

The ethanol fed rats consumed a mean $11 \cdot 2 \pm 1.0 \mathrm{~g}$ (SE) ethanol/kg body weight/day, but gained weight similarly to their pair fed controls which received an isocaloric diet (Fig. 2).

In the ethanol fed rats histological examination of the upper and lower small intestines was normal, and the morphometric measurements of villus height and crypt depth were similar to those in

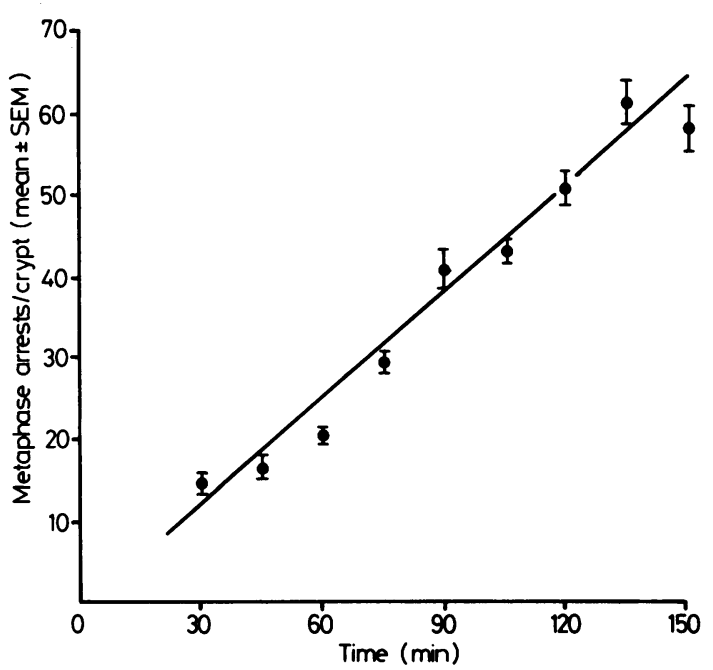

Fig. 1 Typical metaphase arrest curve from the upper small intestine in control rats. Points show the mean number of arrested metaphases in 10 crypts $( \pm S E)$ plotted against time after injection.

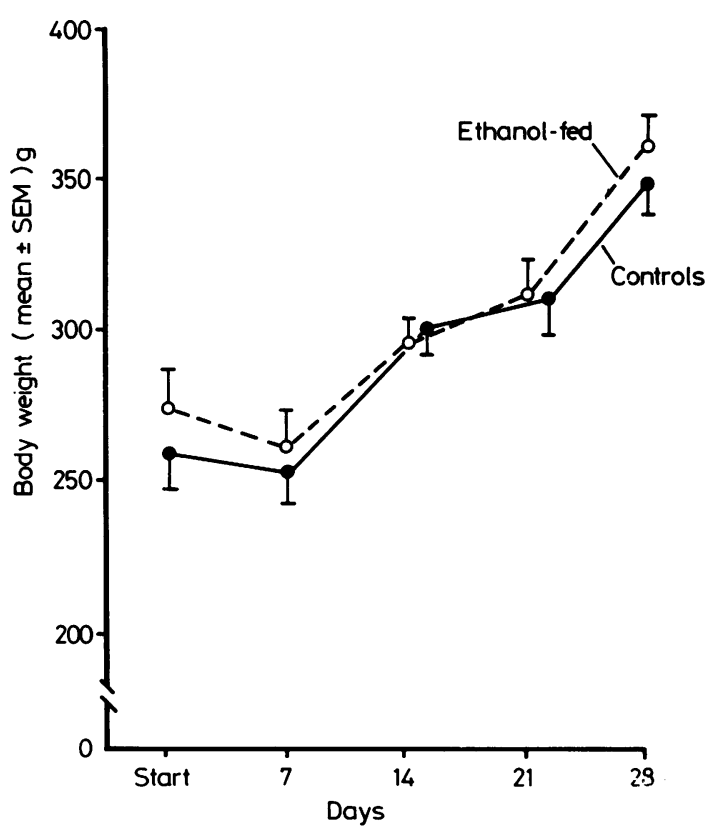

Fig. 2 Body weight in ethanol fed and pair fed control rats. 
control rats (Fig. 3). Also, there was no significant difference between the crypt:villus (C/V) ratios in ethanol fed and control rats (Fig. 4). The crypt cell production rate (CCPR) was significantly decreased, however, in the ethanol fed rats in both the upper and lower small intestines $(p<0 \cdot() 1)$. The net villus influx, calculated by multiplying the CCPR by

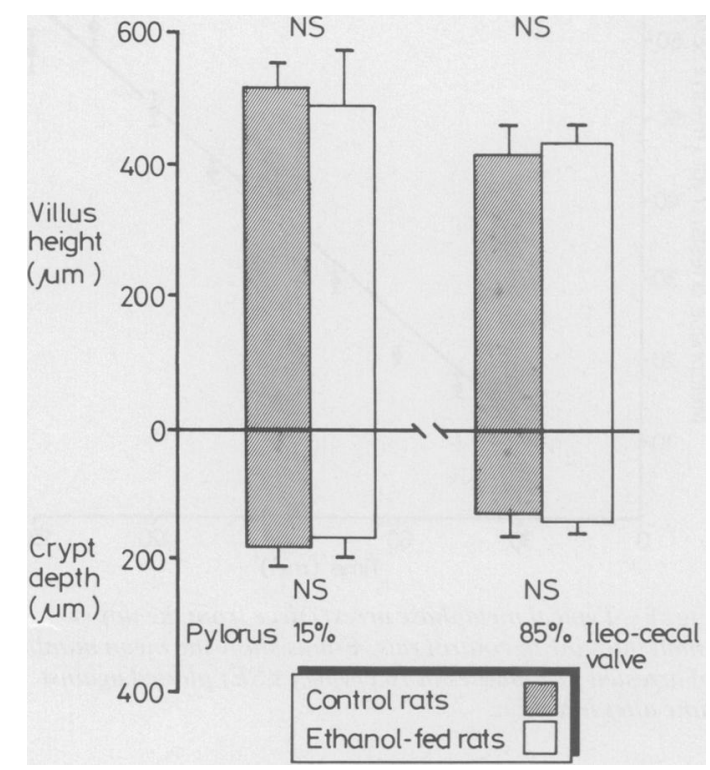

Fig. 3 Morphometric measurements ( $\mu \mathrm{m})$ of the villus height and crypt depth (mean $\pm S E$ ) at $15 \%$ and $85 \%$ of the length of the small intestine from pylorus to ileocaecal valve in control and ethanol fed rats. the $\mathrm{C} / \mathrm{V}$ ratio was also significantly decreased in the ethanol fed rats in both the upper small intestines $(\mathrm{p}<0 \cdot 05)$ and in the lower small intestine $(\mathrm{p}<0 \cdot 01)$.

\section{Discussion}

This study shows that chronic ethanol consumption in the rat is associated with a significant decrease in enterocyte turnover in the small intestine, though the mucosa appears histologically normal. The latter finding is in agreement with reports of normal small intestinal histology in chronic alcoholics on a nutritionally adequate diet ${ }^{11}$ and in some previous studies in chronically ethanol fed rats, ${ }^{12}$ but contrasts with other studies where shortened villi have been described after chronic ethanol consumption both in man and in experimental animals. "It has been suggested that the reduction in height of jejunal villi of chronic alcoholics is caused by an associated folate deficiency rather than by ethanol itself, ' as severe folate deficiency is known to produce villus shortening, decreased mitosis in the crypts, and enlargement of epithelial cell nuclei. ${ }^{18}$ Similarly in the study in rats where chronic ethanol consumption was reported to cause villus shortening. ${ }^{10}$ the weight gain in ethanol fed animals was less than in controls suggesting that the ethanol fed rats were relatively malnourished because of either malabsorption, or a nutritionally inadequate diet. In contrast in the present study weight gain was similar in ethanol fed and control animals. This difference in nutrition may explain the different small bowel histology in the two studies.

Despite the normal histology of the small intestine, the present study shows that enterocyte turn-
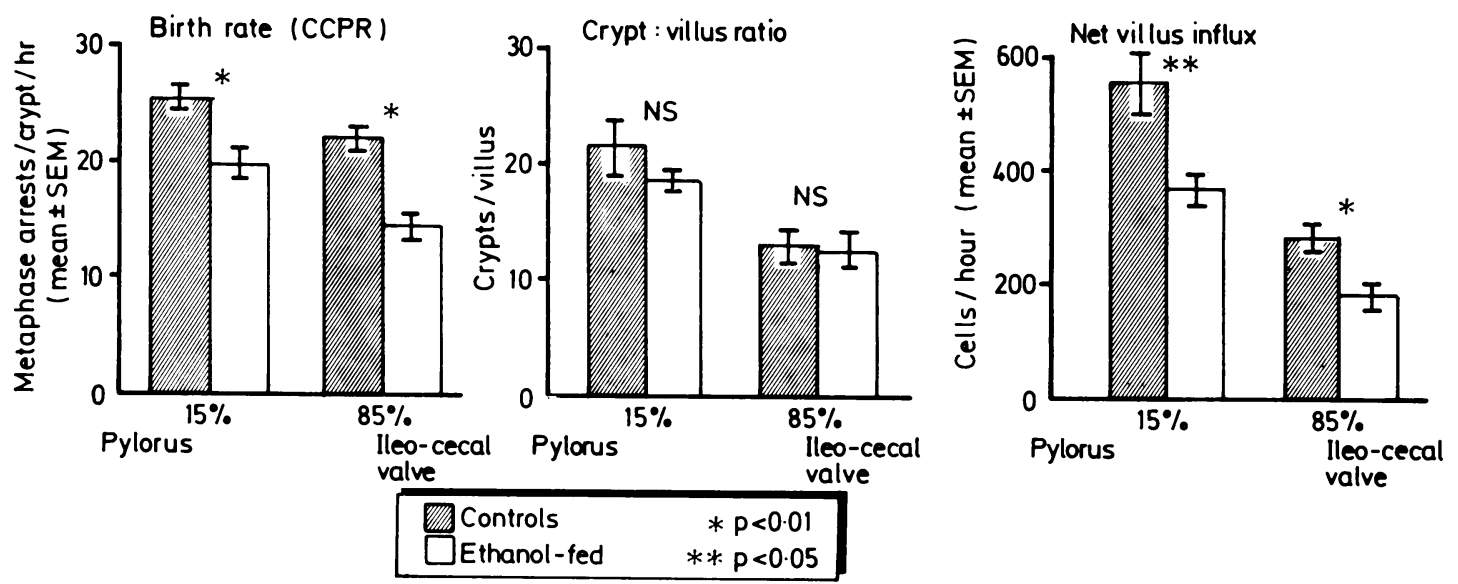

Fig. 4 Crypt cell production rate, crypt:villus ratio and net villus influx (mean $\pm S E$ ) at $15 \%$ and $85 \%$ of the length of the small intestine from pylorus to ileocaecal valve in control and ethanol fed rats. 
over there is significantly decreased by chronic ethanol consumption. Other conditions where changes in enterocyte turnover in the small bowel are known to occur include starvation ${ }^{19} 20$ and after cytotoxic therapy ${ }^{21}$ where it is decreased, and in coeliac disease $\mathrm{e}^{22}$ where it is increased. As the reduction in CCPR was similar in the upper and lower small bowel, this effect of ethanol is probably systemic rather than local, because the intraluminal ethanol concentration falls progressively along the small bowel. ${ }^{23}$

Whether the reduction in small intestinal enterocyte turnover was associated with any impairment of absorptive function in this study is unknown, but it remains a possibility because enterocytes mature functionally as they cross the villus. There was no evidence of obvious malnutrition in the ethanol fed rats, however, which appeared normal and gained weight similar to controls. If absorption was impaired in the ethanol fed animals, there may have been sufficient reserve of small intestinal function to compensate. In chronic alcoholics on a nutritionally poor diet the effect of ethanol reducing enterocyte turnover may further impair the absorptive function of the small intestine and exacerbate malnutrition.

Supported by a grant from the Wellcome Trust. We thank Mrs B Davidson for preparation of the liquid diets, and Prof N A Wright and Dr R A Goodlad for advice.

\section{References}

1 Langman JS, Bell GD. Alcohol and the gastrointestinal tract. $\mathrm{Br}$ Med Bull 1982; 82: 71-5.

2 Tomasulo PA, Kater RMH, Iber FL. Impairment of thiamine absorption in alcoholism. Am J Clin Nutr 1968; 21: 1341-4.

3 Balaghi M, Neal RA. Effect of chronic ethanol administration on thiamine metabolism in the rat. $J$ Nutr 1977; 107: 2144-52.

4 Lindenbaum J, Lieber CS. Alcohol induced malabsorption of vitamin B12 in man. Nature (Lond) 1969; 224: 806.

5 Halsted $\mathrm{CH}$, Griggs RC, Harris JW. The effect of alcoholism on the absorption of folic ( $\mathrm{H}^{3}-\mathrm{PGA}$ ) evaluated by plasma levels and urine excretion. $J$ Lab Clin Med 1967; 69: 116-31.

6 Ghirardi P, Marzo A, Sardini D et al. Changes in intestinal absorption of glucose in rats treated with ethanol. Experientia 1971; 27: 61-2.
7 Kuo YJ, Shanbour LL. Effects of ethanol on sodium, 3-0-methylglucose and 1-alanine transport in the jejunum. Am J Dig Dis 1978; 23: 51-6.

8 Israel Y, Salazar I, Rosenmann E. Inhibitory effects of alcohol on intestinal amino acid transport in vivo and in vitro. J Nutr 1968; 96: 499-504.

9 Jacobs FA, Overvold C. Inhibition of leucine absorption by ethanol. Fed Proc 1976; 35: 463.

10) Baraona E, Romano C. Pirola RC, Lieber CS. Small intestine damage and changes in cell population produced by ethanol ingestion in rat. Gastroenterology 1974; 66: 226-34.

11 Krasner N, Cochran KM, Russell RI et al. Alcohol and absorption from the small intestine. Gut 1976; 17: 245-8.

12 Rubin E, Rybak BJ, Lindenbaum J et al. Ultrastructural changes in the small intestine induced by ethanol. Gastroenterology 1972; 63: 801-14.

13 Worthington BS, Meserole L, Syrotuck JA. Effect of daily ethanol ingestion on intestinal permeability to macromolecules. Am J Dig Dis 1978; 28: 23-32.

14 Draper LR, Gyure LA, Hall JG, Robertson D. Effect of alcohol on the integrity of the intestinal epithelium. Gut 1983; 24: 399-404.

15 Bjarnason I, Ward K, Peters TJ. The leaky gut of alcoholism: possible route of entry for toxic compounds. Lancet 1984; i: 179-82.

16 Ryle PR, Broillet A, Perrisoud D et al. A comparative study of the effects of (+)-catechin and 3-palmitoyl(+)-catechin on alcoholic fatty liver in the rat. Alcohol Alcoholism 1983; 18: 239-48.

17 Goodlad RA, Wright NA. Quantitative studies on epithelial replacement in the gut. Techniques in the Life Sciences, P2. Dig Physiol 1982; P212: 1-23.

18 Hermos JA, Adams WH, Liu YK et al. Mucosa of the small intestine in folate-deficient alcoholics. Ann Intern Med 1972; 76: 957-65.

19 Clarke RM. The effect of growth and fasting on the number of villi and crypts in the small intestine of the albino rat. J Anat 1972; 112: 27-33.

20 Adelwachi HS, Wright NA, Appleton DR, Watson AJ. The effect of starvation and refeeding on cell population kinetics in the rat small bowel mucosa. $J$ Anat 1975; 119: 105-21.

21 Trier JS, Browning TH. Morphologic response of the mucosa of the human small intestine to $\mathrm{x}$-ray exposure. $J$ Clin Invest 1966; 45: 194-204.

22 Watson AJ, Appleton DR, Wright NA. Adaptive cell-proliferation changes in the small intestinal mucosa in coeliac disease. Scand J Gastroenterol 1982; 17: suppl. 74: 115-27.

23 Halsted CH, Robles EA, Mezey E. Distribution of ethanol in the human gastrointestinal tract. Am J Clin Nutr 1972; 26: 831-4. 